PROCEEDINGS OF THE

AMERICAN MATHEMATICAL SOCIETY

Volume 126, Number 5, May 1998, Pages 1425-1428

S 0002-9939(98)04371-8

\title{
A COUNTEREXAMPLE TO A QUESTION OF R. HAYDON, E. ODELL AND H. ROSENTHAL
}

\author{
G. ANDROULAKIS
}

(Communicated by Dale Alspach)

\begin{abstract}
We give an example of a compact metric space $K$, an open dense subset $U$ of $K$, and a sequence $\left(f_{n}\right)$ in $C(K)$ which is pointwise convergent to a non-continuous function on $K$, such that for every $u \in U$ there exists $n \in \mathbf{N}$ with $f_{n}(u)=f_{m}(u)$ for all $m \geq n$, yet $\left(f_{n}\right)$ is equivalent to the unit vector basis of the James quasi-reflexive space of order 1 . Thus $c_{0}$ does not embed isomorphically in the closed linear span $\left[f_{n}\right]$ of $\left(f_{n}\right)$. This answers in the negative a question asked by H. Haydon, E. Odell and H. Rosenthal.
\end{abstract}

\section{INTRODUCTION}

A result of J. Elton [E], which was also proved later by R. Haydon, E. Odell and H. Rosenthal [HOR], states that if $K$ is a compact metric space, and $\left(f_{n}\right)$ is a uniformly bounded sequence in $C(K)$ such that

$$
\sum_{n=1}^{\infty}\left|f_{n+1}(k)-f_{n}(k)\right|<\infty, \forall k \in K,
$$

and the pointwise limit of $\left(f_{n}\right)$ on $K$ is a non-continuous function, then $c_{0}$ embeds isomorphically in the closed linear span $\left[f_{n}\right]$ of $\left(f_{n}\right)$. Thus the following question was naturally raised by R. Haydon, E. Odell and H. Rosenthal:

Question 4.7 in [HOR]. Let $K$ be a compact metric space, $R$ be a residual subset of $K$ (i.e. $K \backslash R$ is a first category set), and $\left(f_{n}\right)$ be a sequence in $C(K)$ which converges pointwise on $K$ to a non-continuous function, and

$$
\sum_{n=1}^{\infty}\left|f_{n+1}(r)-f_{n}(r)\right|<\infty, \text { for all } r \in R .
$$

Does $c_{0}$ embed in the closed linear span $\left[f_{n}\right]$ of $\left(f_{n}\right)$ ?

We will construct a compact metric space $K$, an open dense subset $U$ of $K$ and a sequence $\left(g_{n}\right) \subset C(K)$ such that

(a) $\left(\sum_{i=1}^{n} g_{i}\right)_{n}$ is a uniformly bounded and pointwise convergent sequence on $K$ to a non-continuous function;

(b) For every $u \in U$ there exists $n \in \mathbf{N}$ such that $g_{m}(u)=0$ for every $m \geq n$;

Received by the editors October 19, 1996.

1991 Mathematics Subject Classification. Primary 46B25.

This work is part of the author's Ph.D. thesis, which was completed at the University of Texas at Austin in August 1996 under the supervision of Professor H. Rosenthal.

(C)1998 American Mathematical Society 
(c) $\left[g_{n}\right]$ is isomorphic to the James quasi-reflexive of order 1 space $J$.

Since, of course, $c_{0}$ does not embed isomorphically in $J$, this answers in the negative Question 4.7 of [HOR]. Our construction is very elementary and explicit, even though a shorter proof of the existence of a counterexample to Question 4.7 of $[\mathrm{HOR}]$ can be given along similar lines using more advanced machinery.

\section{The COnstruction}

We recall the definition of the James space $J$ and some simple facts. Let $c_{00}$ denote the finitely supported sequences of real numbers. For $\left(x_{n}\right) \in c_{00}$ we define

$$
\begin{aligned}
\left\|\left(x_{n}\right)\right\|_{J}=\sup \left\{\left[x_{p_{1}}^{2}+\left(x_{p_{2}}-x_{p_{1}}\right)^{2}+\cdots+\left(x_{p_{k}}-x_{p_{k-1}}\right)^{2}\right]^{1 / 2}:\right. \\
\left.k \in \mathbf{N}, \mathbf{1} \leq \mathbf{p}_{\mathbf{1}}<\mathbf{p}_{\mathbf{2}}<\cdots<\mathbf{p}_{\mathbf{k}-\mathbf{1}}<\mathbf{p}_{\mathbf{k}}\right\} .
\end{aligned}
$$

Then the James space $J$ is the completion of $\left(c_{00},\|\cdot\|_{J}\right)$. If $\left(e_{n}\right)$ is the unit vector basis of $c_{00}$, then $\left(e_{n}\right)$ becomes the unit vector basis of $J$, which is monotone and shrinking. Also, $\left(\sum_{i=1}^{n} e_{n}\right)_{n}$ is a weak-Cauchy sequence which is not weakly convergent in $J$. If $\left(a_{n}\right) \in c_{0}$ is a monotone sequence of real numbers (i.e. nonincreasing, or non-decreasing) then $\left\|\left(a_{n}\right)\right\|_{J}=\left|a_{1}\right|$ (this is because if $a, b \in \mathbf{R}$ with $a b \geq 0$, then $\left.a^{2}+b^{2} \leq(a+b)^{2}\right)$.

Notation. For $\left(a_{n}\right),\left(b_{n}\right) \in c_{00}$, we define $\left(a_{n}\right) \cdot\left(b_{n}\right) \in c_{00}$ by

$$
\left(a_{n}\right) \cdot\left(b_{n}\right)=\left(a_{n} b_{n}\right) \text {. }
$$

Lemma 2.1. For $\left(a_{n}\right),\left(b_{n}\right) \in c_{00}$ we have

$$
\left\|\left(a_{n}\right) \cdot\left(b_{n}\right)\right\|_{J} \leq\left\|\left(a_{n}\right)\right\|_{J}\left\|\left(b_{n}\right)\right\|_{\infty}+\left\|\left(a_{n}\right)\right\|_{\infty}\left\|\left(b_{n}\right)\right\|_{J} .
$$

Proof. For some $k \in \mathbf{N}$ and some finite sequence of positive integers $1 \leq p_{1}<p_{2}<$ $\cdots p_{k}$ we have

$$
\begin{aligned}
\left\|\left(a_{n}\right) \cdot\left(b_{n}\right)\right\|_{J}= & {\left[\left(a_{p_{1}} b_{p_{1}}\right)^{2}+\left(a_{p_{2}} b_{p_{2}}-a_{p_{1}} b_{p_{1}}\right)^{2}+\cdots+\left(a_{p_{k}} b_{p_{k}}-a_{p_{k-1}} b_{p_{k-1}}\right)^{2}\right]^{1 / 2} } \\
= & {\left[\left(a_{p_{1}} b_{p_{1}}\right)^{2}+\left(a_{p_{2}}\left(b_{p_{2}}-b_{p_{1}}\right)+\left(a_{p_{2}}-a_{p_{1}}\right) b_{p_{1}}\right)^{2}\right.} \\
& \left.+\cdots+\left(a_{p_{k}}\left(b_{p_{k}}-b_{p_{k-1}}\right)+\left(a_{p_{k}}-a_{p_{k-1}}\right) b_{p_{k-1}}\right)^{2}\right]^{1 / 2} .
\end{aligned}
$$

Therefore by the triangle inequality in $\ell_{2}$ we have that

$$
\begin{aligned}
\left\|\left(a_{n}\right) \cdot\left(b_{n}\right)\right\|_{J} \leq & {\left[a_{p_{1}}^{2} b_{p_{1}}^{2}+\left(a_{p_{2}}-a_{p_{1}}\right)^{2} b_{p_{1}}^{2}+\cdots+\left(a_{p_{k}}-a_{p_{k-1}}\right)^{2} b_{p_{k-1}}^{2}\right]^{1 / 2} } \\
& +\left[a_{p_{2}}^{2}\left(b_{p_{2}}-b_{p_{1}}\right)^{2}+\cdots+a_{p_{k}}^{2}\left(b_{p_{k}}-b_{p_{k-1}}\right)^{2}\right]^{1 / 2} \\
\leq & {\left[a_{p_{1}}^{2}+\left(a_{p_{2}}-a_{p_{1}}\right)^{2}+\cdots+\left(a_{p_{k}}-a_{p_{k-1}}\right)^{2}\right]^{1 / 2}\left\|\left(b_{n}\right)\right\|_{\infty} } \\
& +\left\|\left(a_{n}\right)\right\|_{\infty}\left[\left(b_{p_{2}}-b_{p_{1}}\right)^{2}+\cdots+\left(b_{p_{k}}-b_{p_{k-1}}\right)^{2}\right]^{1 / 2} \\
\leq & \left\|\left(a_{n}\right)\right\|_{J}\left\|\left(b_{n}\right)\right\|_{\infty}+\left\|\left(a_{n}\right)\right\|_{\infty}\left\|\left(b_{n}\right)\right\|_{J},
\end{aligned}
$$

which finishes the proof of the lemma.

Now we are ready to see the counterexample. Let $K:=\left\{(a, b) \in \mathbf{R}^{\mathbf{2}}: \mathbf{0} \leq \mathbf{a} \leq\right.$ $\mathbf{1}, \mathbf{0} \leq \mathbf{b} \leq \mathbf{1}\}$. Since $C[0,1]$ is universal for the class of separable spaces, there exist a sequence $\left(f_{n}\right) \subset C[0,1]$ and $M>0$ such that $\left(f_{n}\right)$ is $M$-equivalent to the unit vector basis of $J$. For $n \in \mathbf{N}$ set $K_{n}:=\left\{(a, b) \in \mathbf{R}^{\mathbf{2}}: \mathbf{0} \leq \mathbf{a} \leq \mathbf{1}, \mathbf{1} / \mathbf{2}^{\mathbf{n}} \leq \mathbf{b} \leq \mathbf{1}\right\}$, $L_{n}:=\left\{(a, b) \in \mathbf{R}^{\mathbf{2}}: \mathbf{0} \leq \mathbf{a} \leq \mathbf{1}, \mathbf{b}=\mathbf{1} / \mathbf{2}^{\mathbf{n}}\right\}, L:=\{(a, 0): 0 \leq a \leq 1\}$ and $U=K \backslash L$. Now, for $n \in \mathbf{N}$ define $g_{n}: K \rightarrow \mathbf{R}$ by

- $g_{n} \mid K_{n} \equiv 0$, 
- for every $0 \leq a \leq 1, g_{n}$ restricted to the segment connecting the points $\left(a, 1 / 2^{n}\right)$ and $(a, 0)$, is linear,

- $g_{n} \mid L \equiv f_{n}$,

- $g_{n}$ is continuous on $K$.

We will show that $\left(g_{n}\right)$ is equivalent to the unit vector basis $\left(e_{i}\right)$ of the James space. This will imply that $\left(\sum_{i=1}^{n} g_{i}\right)_{n}$ is a weak Cauchy sequence which is not weakly convergent, which will finish the proof. Let $n \in \mathbf{N}$ and $\left(\lambda_{i}\right)_{i=1}^{n} \subset \mathbf{R}$. We want to estimate $\left\|\lambda_{1} g_{1}+\cdots+\lambda_{n} g_{n}\right\|_{\infty}$. For $(a, b),(c, d) \in K$, let $[(a, b),(c, d)]$ denote the linear segment connecting the points $(a, b)$ and $(c, d)$. For every $0 \leq a \leq 1$ we have that

- $\left(\lambda_{1} g_{1}+\cdots+\lambda_{n} g_{n}\right) \mid[(a, 1),(a, 1 / 2)] \equiv 0$,

- $\left(\lambda_{1} g_{1}+\cdots+\lambda_{n} g_{n}\right) \mid\left[\left(a, 1 / 2^{i}\right),\left(a, 1 / 2^{i+1}\right)\right]$ is linear, for every $i=1, \ldots, n-1$,

- $\left(\lambda_{1} g_{1}+\cdots+\lambda_{n} g_{n}\right) \mid\left[\left(a, 1 / 2^{n}\right),(a, 0)\right]$ is linear,

- $\lambda_{1} g_{1}+\cdots+\lambda_{n} g_{n}$ is continuous on $K$.

Therefore we obtain

$$
\begin{aligned}
\| \lambda_{1} g_{1}+ & \cdots+\lambda_{n} g_{n} \|_{\infty} \\
& =\max _{2 \leq k \leq n}\left\|\left(\lambda_{1} g_{1}+\cdots+\lambda_{n} g_{n}\right)\left|L_{k}\left\|_{\infty} \vee\right\|\left(\lambda_{1} g_{1}+\cdots+\lambda_{n} g_{n}\right)\right| L\right\|_{\infty} \\
& =\max _{2 \leq k \leq n}\left\|\left(\lambda_{1} g_{1}+\cdots \lambda_{k-1} g_{k-1}\right) \mid L_{k}\right\|_{\infty} \vee\left\|\lambda_{1} f_{1}+\cdots+\lambda_{n} f_{n}\right\|_{\infty} .
\end{aligned}
$$

Therefore we immediately obtain the lower estimate

$$
\begin{aligned}
\left\|\lambda_{1} g_{1}+\cdots+\lambda_{n} g_{n}\right\|_{\infty} & \geq\left\|\lambda_{1} f_{1}+\cdots+\lambda_{n} f_{n}\right\|_{\infty} \\
& \geq \frac{1}{M}\left\|\lambda_{1} e_{1}+\cdots+\lambda_{n} e_{n}\right\|_{J} .
\end{aligned}
$$

For the upper estimate we need to estimate $\left\|\left(\lambda_{1} g_{1}+\cdots+\lambda_{n} g_{n} \mid L_{k}\right)\right\|_{\infty}$ for $2 \leq$ $k \leq n$. Note that for $0 \leq a \leq 1$ and $2 \leq k \leq n$ we have

$$
\begin{aligned}
\left(\lambda_{1} g_{1}+\right. & \left.\cdots+\lambda_{n} g_{n}\right)\left(a, 1 / 2^{k}\right) \\
& =\lambda_{1} \frac{\frac{1}{2}-\frac{1}{2^{k}}}{\frac{1}{2}} f_{1}(a)+\lambda_{2} \frac{\frac{1}{2^{2}}-\frac{1}{2^{k}}}{\frac{1}{2^{2}}} f_{2}(a)+\cdots+\lambda_{k-1} \frac{\frac{1}{2^{k-1}}-\frac{1}{2^{k}}}{\frac{1}{2^{k-1}}} f_{k-1}(a) \\
& =\lambda_{1} \frac{2^{k-1}-1}{2^{k-1}} f_{1}(a)+\lambda_{2} \frac{2^{k-2}-1}{2^{k-2}} f_{2}(a)+\cdots+\lambda_{k-1} \frac{2-1}{2} f_{k-1}(a) .
\end{aligned}
$$

Therefore we have that

$$
\begin{aligned}
\| \lambda_{1} g_{1}+ & \cdots+\lambda_{k-1} g_{k-1} \mid L_{k} \|_{\infty} \\
= & \left\|\lambda_{1} \frac{2^{k-1}-1}{2^{k-1}} f_{1}+\lambda_{2} \frac{2^{k-2}-1}{2^{k-2}} f_{2}+\cdots+\lambda_{k-1} \frac{2-1}{2} f_{k-1}\right\|_{\infty} \\
\leq & M\left\|\lambda_{1} \frac{2^{k-1}-1}{2^{k-1}} e_{1}+\lambda_{2} \frac{2^{k-2}-1}{2^{k-2}} e_{2}+\cdots+\lambda_{k-1} \frac{2-1}{2} e_{k-1}\right\|_{J} \\
= & M \|\left(\lambda_{1}, \lambda_{2}, \cdots, \lambda_{k-1}, 0, \ldots\right) \\
& \quad \cdot\left(\frac{2^{k-1}-1}{2^{k-1}}, \frac{2^{k-2}-1}{2^{k-2}}, \ldots, \frac{2-1}{2}, 0, \ldots\right) \|_{J} \\
\leq & M\left\|\lambda_{1} e_{1}+\cdots+\lambda_{k-1} e_{k-1}\right\|_{J} \cdot 1 \\
& +M\left\|\left(\lambda_{i}\right)_{i=1}^{k-1}\right\|_{\infty}\left\|\left(\frac{2^{k-1}-1}{2^{k-1}}, \ldots, \frac{2-1}{2}, 0, \ldots\right)\right\|_{J} \quad \text { (by Lemma 2.1) }
\end{aligned}
$$




$$
\begin{aligned}
\leq & M\left\|\lambda_{1} e_{1}+\cdots+\lambda_{k-1} e_{k-1}\right\|_{J}+M\left\|\left(\lambda_{i}\right)\right\|_{\infty} \frac{2^{k-1}-1}{2^{k-1}} \quad \text { (since the } \\
& \text { sequence }\left(\frac{2^{k-1}-1}{2^{k-1}}, \frac{2^{k-2}-1}{2^{k-2}}, \ldots, \frac{2-1}{2}, 0, \ldots,\right) \text { is decreasing) } \\
\leq & 2 M\left\|\lambda_{1} e_{1}+\cdots+\lambda_{k-1} e_{k-1}\right\|_{J} \quad\left(\text { since }\left\|\left(\lambda_{i}\right)_{i=1}^{k-1}\right\|_{\infty} \leq\left\|\left(\lambda_{i}\right)_{i=1}^{k-1}\right\|_{J}\right) .
\end{aligned}
$$

Also, since $\left\|\lambda_{1} f_{1}+\cdots+\lambda_{n} f_{n}\right\|_{J} \leq M\left\|\lambda_{1} e_{1}+\cdots+\lambda_{n} e_{n}\right\|_{J}$, we obtain

$$
\left\|\lambda_{1} g_{1}+\cdots+\lambda_{n} g_{n}\right\|_{\infty} \leq 2 M\left\|\lambda_{1} e_{1}+\cdots+\lambda_{n} e_{n}\right\|_{J}
$$

This finishes the proof.

\section{REFERENCES}

[E] J. Elton, Extremely weakly unconditionally convergent series, Israel J. Math. 40 (1981), 255-258. MR 83e:46015

[HOR] R. Haydon, E. Odell, H. Rosenthal, On certain classes of Baire-1 functions with applications to Banach space theory, Lecture Notes in Mathematics Vol. 1470, Springer-Verlag, Berlin 1991. MR 92h:46018

Department of Mathematics, University of Missouri-Columbia, Columbia, Missouri 65211

E-mail address: giorgis@math.missouri.edu 